\title{
Synthesis of sandwich-like molybdenum sulfide/mesoporous organosilica nanosheets for photo-thermal conversion and stimuli-responsive drug release
}

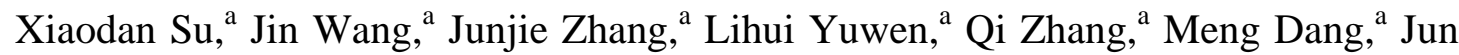
Tao, ${ }^{a}$ Xiaobo Ma, ${ }^{*, b}$ Shouju Wang, ${ }^{*, c}$ and Zhaogang Teng ${ }^{*, c}$

${ }^{\text {a }}$ Key Laboratory for Organic Electronics \& Information Displays and Institute of Advanced Materials, Nanjing University of Posts and Telecommunications (NUPT), Nanjing 210046, P.R. China.

${ }^{\mathrm{b}}$ School of Optoelectronic Engineering, Nanjing University of Posts and Telecommunications (NUPT), Nanjing 210023, P.R. China.

${ }^{c}$ Department of Medical Imaging, Jinling Hospital, School of Medicine, Nanjing University, Nanjing 210002, P.R. China

\begin{abstract}
Sandwich-like molybdenum sulfide/mesoporous organosilica nanosheets (denoted as $\mathrm{MoS}_{2} @ \mathrm{MOS}$ ) have been prepared for the first time via direct growth of ethane-bridged mesostructured organosilica on $\mathrm{MoS}_{2}$ nanosheets by using cetyltrimethylammonium bromide (CTAB) as structure directing agent. The obtained $\mathrm{MoS}_{2} @ \mathrm{MOS}$ nanosheets possess well-defined sandwich-like structure, high surface
\end{abstract}

\footnotetext{
* Corresponding authors

E-mail addresses: maxiaobo@njupt.edu.cn (X.Ma), Shouju.wang@gmail.com (S. Wang), and zhaogangteng@163.com (Z. Teng).
} 
area $\left(\sim 920 \mathrm{~cm}^{2} / \mathrm{g}\right)$, uniform pore size $(\sim 4.2 \mathrm{~nm})$, large pore volume $\left(\sim 1.41 \mathrm{~cm}^{3} \mathrm{~g}^{-1}\right)$. In vitro cytotoxicity assessments demonstrate that the $\mathrm{MoS}_{2} @ \mathrm{MOS}$ nanosheets have excellent biocompatibility. Owing to the encapsulation of the $\mathrm{MoS}_{2}$, the obtained $\mathrm{MoS}_{2} @ \mathrm{MOS}$ nanosheets have photo-thermal conversion capability and photo-thermally controlled drug release property. These properties make the $\mathrm{MoS}_{2} @ \mathrm{MOS}$ nanosheets promising for biomedical applications.

Keywords: mesoporous organosilica, $\mathrm{MoS}_{2}$, sandwich structure, photo-thermal conversion, drug release

\section{Introduction}

Mesoporous organosilicas have attracted more and more research attention because of their combined advantages of ordered mesostructure, organic and inorganic fragments hybridized frameworks, large specific surface areas, good biocompatibility and extensive promising applications in various areas such as separation, catalysis, and biomedicine. ${ }^{[1-6]}$ Due to the hydrolysis and condensation of the organosilica precursors in solution are highly controllable. Recently, tremendous efforts have been devoted to synthesize functional organosilica composites by coating mesoporous organosilica on magnetic or luminescent particles to extent their properties and applications. ${ }^{[7-11]}$ However, to the best of knowledge, the organosilica based multifunctional materials are limited to core-shell structures with a spherical shape. ${ }^{[12,13]}$ Sheet-like mesoporous organosilica composites have not been reported so 
far.

In recent years, molybdenum sulfide $\left(\mathrm{MoS}_{2}\right)$ nanosheets that resemble the 2D structure of graphene have become a hotspot of research for biomedical applications due to their remarkable photo-thermal effect in an aqueous medium. ${ }^{[14-18]}$ The single- or few-layered $\mathrm{MoS}_{2}$ can be readily produced from bulk $\mathrm{MoS}_{2}$ via lithium intercalation. ${ }^{[19]}$ However, newly synthesized $\mathrm{MoS}_{2}$ generally possesses low stability and easily aggregates in aqueous solution. Therefore, modification of the $\mathrm{MoS}_{2}$ to ensure satisfied stability and good biocompatibility is very important for their biomedical applications. ${ }^{[20-22]}$

Herein, we successfully prepared a sandwich-like structured $\mathrm{MoS}_{2} @ \mathrm{MOS}$ nanosheets by direct co-assembly of cetyltrimethylammonium bromide (CTAB) surfactant and organosilicates on the $\mathrm{MoS}_{2}$. The obtained sandwich-like $\mathrm{MoS}_{2} @ \mathrm{MOS}$ nanosheets possess high surface area $\left(\sim 920 \mathrm{~cm}^{2} / \mathrm{g}\right)$, ordered mesopores $(\sim 4.2 \mathrm{~nm})$, large pore volume $\left(\sim 1.41 \mathrm{~cm}^{3} \mathrm{~g}^{-1}\right)$. Moreover, cytotoxicity and photo-thermal experiments show the $\mathrm{MoS}_{2} @ \mathrm{MOS}$ nanosheets have excellent biocompatibility, photo-thermal conversion capability and photo-thermally controlled drug release properties, showing great potential for biomedical applications.

\section{Experimental}

\subsection{Materials.}

Molybdenum (IV) sulfide $\left(\mathrm{MoS}_{2}\right)$ powder $(<2 \mu \mathrm{m}, 99 \%)$ was purchased from Sigma-Aldrich; n-butyllithium (n-BuLi, 2.4 M hexane solution) was bought from 
Amethyst; CTAB, concentrated ammonia aqueous solution (25 wt \%), and anhydrous ethanol were purchased from Sinopharm Chemical Reagent Co., Ltd. (Shanghai, China). 1,2-Bis(triethoxysilyl)ethane (BTSE) were bought from Sigma-Aldrich (St. Louis, MO, USA). Decane with a purity of $>99 \%$ were purchased from Acros Organics (Merck, Germany). Millipore water was used in all experiments.

\subsection{Characterization.}

Transmission electron microscopy (TEM) images were obtained on an HT7700 microscope (Hitachi, Tokyo, Japan) operated at an accelerating voltage $100 \mathrm{kV}$. Energy dispersive X-ray (EDX) analyses and dark-field scanning TEM (DF-STEM) were performed on a JEOL JEM-2100F electron microscope (200 Kv) equipped with an energy dispersive X-ray spectrometer. X-ray power diffraction (XRD) was measured on a Bruker model D8 focus diffractometer with $\mathrm{Cu} \mathrm{K \alpha}$ radiation $(0.154 \mathrm{~nm})$ operated at $40 \mathrm{kV}$ and $40 \mathrm{~mA}$. Fourier transform infrared (FT-IR) spectra were obtained on a Nicolet NEXUS870 spectrometer (USA), using KBr pellets of the solid samples. Nitrogen sorption isotherms were measured using a Micromeritics Tristar 3000 analyzer at $-196{ }^{\circ} \mathrm{C}$. The samples were degassed at $150{ }^{\circ} \mathrm{C}$ for $10 \mathrm{~h}$ before the measurements. The specific surface area was calculated with the Brunaue-Emmett-Teller (BET) method by using the adsorption data. The pore size distribution was obtained by applying proper nonlocal density functional theory (NLDFT) methods from the adsorption branch of isotherms. The total pore volume was evaluated from the adsorbed amount at a relative pressure $\left(p / p_{0}\right)$ of 0.994 .

\subsection{Preparation of $\mathrm{MoS}_{2}$ nanosheets.}


$\mathrm{MoS}_{2}$ nanosheets were synthesized by lithium intercalation-exfoliation and exfoliation method. ${ }^{[23,24]}$ Briefly, a mixture of $0.2 \mathrm{~g} \mathrm{MoS}_{2}$ and $6.25 \mathrm{~mL} \mathrm{n-BuLi}$ hexane solution was stirred at room temperature for $48 \mathrm{~h}$ under Ar atmosphere, then allowed to settle for several hours. Excess $\mathrm{n}$-BuLi solution in the reaction mixture was removed by a syringe, and the black solid was collected in a tube. Ar saturated water was carefully introduced into the tube to avoid sputtering under the protection of Ar, since lots of hydrogen gas are released at that moment. The $\mathrm{MoS}_{2}$ nanosheets aqueous suspension was sonicated for $1.5 \mathrm{~h}$ to thoroughly complete the exfoliation process and then centrifuged at $1200 \mathrm{rpm}$ for $30 \mathrm{~min}$. After centrifugation, the precipitates were discarded. Further purification by centrifugation was repeated at least two times. Finally, the precipitated $\mathrm{MoS}_{2}$ nanosheets were dispersed in water and the concentration of the $\mathrm{MoS}_{2}$ nanosheets aqueous solution was $1.308 \mathrm{mg} \mathrm{mL}^{-1}$.

\subsection{Preparation of sandwich-like $\mathrm{MoS}_{2} @$ MOS nanosheets.}

The $\mathrm{MoS}_{2} @ \mathrm{MOS}$ nanosheets were prepared by co-assembly of CTAB and ethane-bridged organosilicates on $\mathrm{MoS}_{2}$. In a typical experiment, CTAB (0.0386 g) was dissolved in $11.9 \mathrm{~mL}$ water, $3.3 \mathrm{~mL} \mathrm{MoS}_{2}$ nanosheets aqueous solution (1.308 $\left.\mathrm{mg} \mathrm{mL} \mathrm{m}^{-1}\right), 0.72 \mathrm{~mL}$ of concentrated ammonia aqueous solution (25 wt \%), and 0.12 $\mathrm{mL}$ of decane. The mixture was stirred $20 \mathrm{~min}$ at $50{ }^{\circ} \mathrm{C}$ and a mixed solution containing $0.08 \mathrm{~mL}$ BTSE and $0.32 \mathrm{~mL}$ ethanol was then added. After $12 \mathrm{~h}$, the products were collected by centrifugation and washed with water three times. Finally, CTAB templates were removed from the products by three solvent-extractions in 30 $\mathrm{mL}$ ethanol (containing $0.06 \mathrm{~mL} \mathrm{HCl}$ ) at $60{ }^{\circ} \mathrm{C}$ for $3 \mathrm{~h}$. After washing with ethanol for 
three times and dried under a high vacuum, the sandwich-like $\mathrm{MoS}_{2} @ \mathrm{MOS}$ nanosheets were obtained.

\subsection{Cell viability assay.}

Cell viability is assessed by the MTT method. First, $1 \times 10^{4} \mathrm{MCF}-7$ cells were plated in a 96-well culture plate, then incubated with the $\mathrm{MoS}_{2} @ \operatorname{MOS}$ nanosheets at various concentrations $\left(100,200,400,600,700,800,900\right.$ and $\left.1000 \mu \mathrm{g} \mathrm{mL}^{-1}\right)$ for $24 \mathrm{~h}$ in a humidified incubator. The absorbance at $570 \mathrm{~nm}$ was measured with a microplate reader (BioTek Instruments, Winooski, VT) after dissolving the precipitates with DMSO $(150 \mu \mathrm{L})$. Each experiment was repeated three times, and six wells were treated in parallel for each concentration. The cell viability (\%) was calculated according to our previous work. ${ }^{[25]}$

\subsection{Measurements of photo-thermal property.}

The $\mathrm{MoS}_{2} @ \mathrm{MOS}$ nanosheets dispersed in water with concentrations from 0 to $1000 \mu \mathrm{g} \mathrm{mL}^{-1}$ were irradiated with a power density of 2 or $5 \mathrm{~W} \mathrm{~cm}^{-2}$ for $5 \mathrm{~min}$ from an 808 nm laser system (Hi-Tech Optoelectronics Co., Ltd., China).

\subsection{DOX loading and releasing.}

In the loading experiments, DOX was loaded by mixing various concentrations of drug with $\mathrm{MoS}_{2} @ \mathrm{MOS}$ in PBS (pH 7.4) for 5, 24, 48, and $72 \mathrm{~h}$ at $37{ }^{\circ} \mathrm{C}$. The unloaded DOX was separated by centrifugation at 13,200 rpm for $5 \mathrm{~min}$. The DOX concentration in the supernatant was determined to calculate the loading efficiency and capacity.

In the releasing experiments, $0.5 \mathrm{mg} \mathrm{mL}^{-1} \mathrm{MoS}_{2} @ \mathrm{MOS}$ was mixed with $0.1 \mathrm{mg}$ 
$\mathrm{mL}^{-1}$ DOX in PBS for $72 \mathrm{~h}$. Then the obtained DOX/MoS ${ }_{2} @$ MOS was centrifuged and redispersed in PBS with a concentration of $0.5 \mathrm{mg} \mathrm{mL}^{-1}$. The solution was then irradiated by an $808 \mathrm{~nm}$ laser at power density of $5 \mathrm{~W} \mathrm{~cm}^{-2}$ for $60 \mathrm{~min}$. A sample of the solution was centrifuged every 10 min during the irradiation to determine the DOX concentration in the supernatant and calculate the released percentage of DOX. The releasing behavior of DOX/MoS $@$ @MOS in PBS without irradiation was also measured as control.

\section{Results and discussion}

The preparation process of the sandwich-like $\mathrm{MoS}_{2} @ \mathrm{MOS}$ nanosheets is illustrated in Scheme 1. First, $\mathrm{MoS}_{2}$ nanosheets were prepared via lithium intercalation-exfoliation method. Then the $\mathrm{MoS}_{2}$ nanosheets were dispersed in an aqueous solution containing ammonia, CTAB, and small amount of decane. The CTAB micelles were adsorbed on the $\mathrm{MoS}_{2}$ through electrostatic interaction. It should be pointed out that the introduced decane has a swelling behavior for the CTAB micelles and results in relatively large mesopores. After the organosilica precursor BTSE is added, they are hydrolyzed to form negatively charged oligomeric organosilicate species and co-assemble with the CTAB micelles on the $\mathrm{MoS}_{2}$ nanosheets. With the reaction proceeding, the organosilicates were cross-linked each other on the $\mathrm{MoS}_{2}$ nanosheets, and the CTAB-organosilicate composites grow gradually and form ordered mesostructures. After removal of surfactants by solvent extraction, sandwich-like $\mathrm{MoS}_{2} @ \mathrm{MOS}$ nanosheets with $\mathrm{MoS}_{2}$ located in interlayer 
and mesoporous organosilica at upper and lower layers are finally obtained.

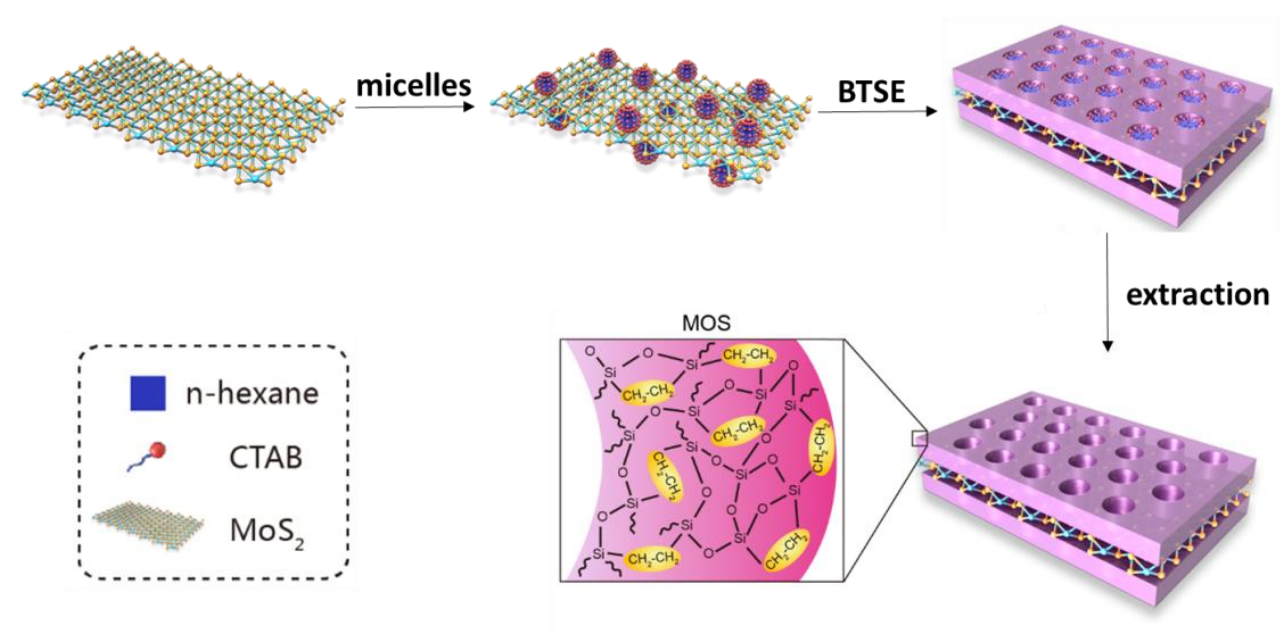

Scheme 1. Illustration of the preparation process of the sandwich-like $\mathrm{MoS}_{2} @ \mathrm{MOS}$ nanosheets.

TEM images show that the obtained $\mathrm{MoS}_{2} @ \mathrm{MOSs}$ possess sheet structure and excellent dispersibility in aqueous solution owing to the coating of the ethane-bridged mesoporous organosilica (Figure 1A). In contrast, the naked $\mathrm{MoS}_{2}$ aggregates easily in aqueous solution (Figure S1). Also, TEM observation demonstrates that no free PMO nanoparticles or naked $\mathrm{MoS}_{2}$ nanosheets are presented. The $\mathrm{MoS}_{2} @ \mathrm{MOS}$ nanosheets have an average lateral size of $200 \mathrm{~nm}$. The DF-STEM image shows that the $\mathrm{MoS}_{2} @ \mathrm{MOS}$ nanosheets have well-defined sandwich-like structure (Figure 1B). High magnification TEM image further indicates the mesopores are oriented to surface of the $\mathrm{MoS}_{2}$ nanosheets (Figure 1C). Top-view TEM image further demonstrates the radially oriented mesopores with openings at surface (Figure 1D). The radially oriented mesochannels favor the adsorption and release of guest 
molecules, such as anticancer drugs. EDX line scanning (Figure 2) clearly reveals the sandwich-like structure with $\mathrm{MoS}_{2}$ sheets between the organosilica layers. The integrated energy dispersive X-ray spectroscopy (EDX) analysis further confirms the presence of Si, O, S, and Mo (Figure S2).
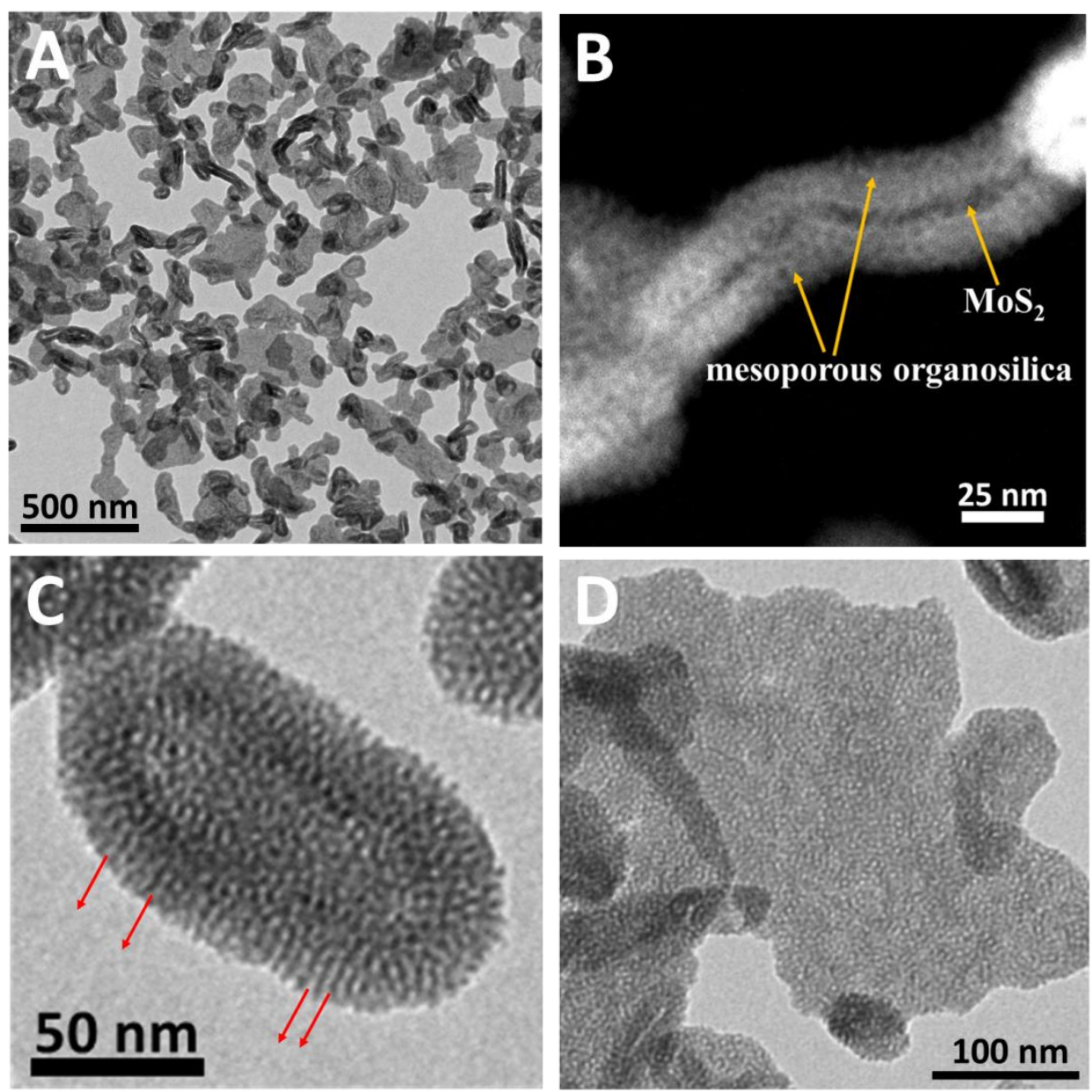

Figure 1. (A, C, D) TEM images and (B) DF-STEM of the sandwich-like $\mathrm{MoS}_{2} @$ MOS nanosheets prepared by co-assembly of CTAB micelles with hydrolyzed BTSE on $\mathrm{MoS}_{2}$. 

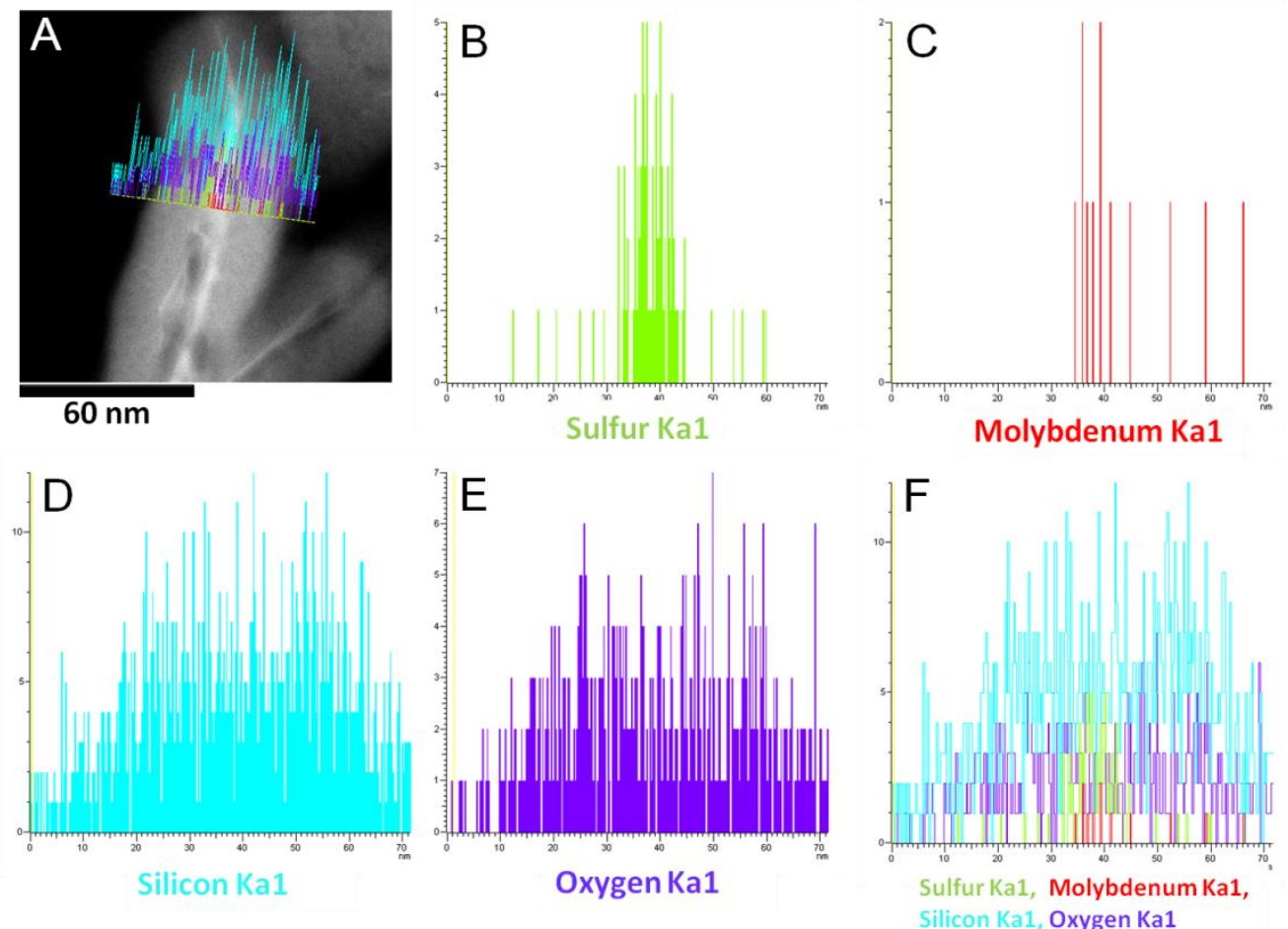

Figure 2. (B-F) EDX line scanning profiles of $\mathrm{S}, \mathrm{Mo}, \mathrm{Si}$, and $\mathrm{O}$ recorded from the $\mathrm{MoS}_{2} @$ MOS nanosheets shown in (A).

The XRD pattern of the sandwich-like $\mathrm{MoS}_{2} @$ MOS nanosheets show an obvious peak at $1.58^{\circ}$ (Figure 3A), suggesting a ordered mesostructure. ${ }^{[26,27]}$ The FT-IR spectrum of the $\mathrm{MoS}_{2} @ \mathrm{MOS}$ nanosheets display the stretch vibrations of the $\mathrm{C}-\mathrm{H}$ bond around $2896 \mathrm{~cm}^{-1}$ and bending vibration of the $\mathrm{C}-\mathrm{H}$ bond in the $-\mathrm{CH}_{2}-\mathrm{CH}_{2}-$ moieties at $1412 \mathrm{~cm}^{-1}$ (Figure 3B), which suggests that the ethane-bridged organosilica are successfully deposited on the $\mathrm{MoS}_{2}$ nanosheets. ${ }^{[28,29]}$ Nitrogen sorption isotherms of the $\mathrm{MoS}_{2} @ \mathrm{MOS}$ nanosheets show a type IV curve with capillary condensation at relative pressures of $0.5-0.85$ and $0.85-1.0$ (Figure 3C), suggesting typical mesoporous structure. The surface area and pore volume are calculated to be as high as $920 \mathrm{~m}^{2} \mathrm{~g}^{-1}$ and $1.41 \mathrm{~cm}^{3} \mathrm{~g}^{-1}$, respectively. The high surface 
area and large pore volume is attributed to the coating of the mesoporous organosilica layer. The pore size distribution calculated based on the nonlocal density functional theory (NLDFT) reveals that the $\mathrm{MoS}_{2} @ \mathrm{MOS}$ nanosheets have a uniform pore size of about $4.2 \mathrm{~nm}$ (Figure 3D). The yield of the $\mathrm{MoS}_{2} @ \mathrm{MOS}$ nanosheets with the size of $200 \mathrm{~nm}$ is $71 \%$.
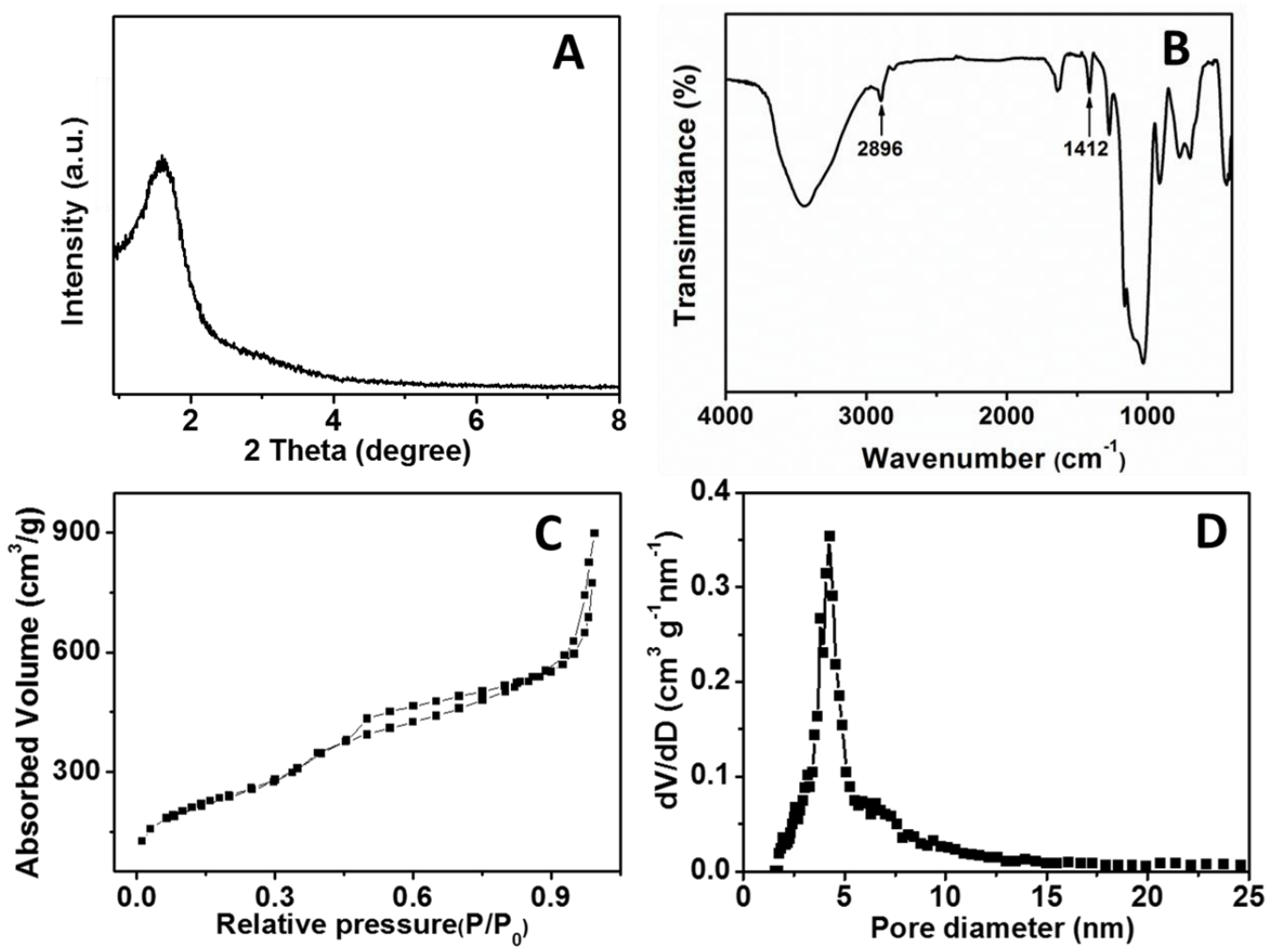

Figure 3. (A) XRD, (B) FT-IR spectrum, (C) Nitrogen sorption isotherms and (D) pore size distribution curve of the sandwich-like $\mathrm{MoS}_{2} @ \mathrm{MOS}$ nanosheets.

The biocompatibility of the $\mathrm{MoS}_{2} @$ MOS nanosheets were further assessed with MCF-7 cell. It is observed that the cell viability is retained about $100 \%$ when they are incubated with the $\mathrm{MoS}_{2} @ \mathrm{MOS}$ nanosheets at concentrations ranging from 100 to $1000 \mu \mathrm{g} \mathrm{mL} \mathrm{m}^{-1}$ for $24 \mathrm{~h}$ (Figure 4). The results demonstrate that the $\mathrm{MoS}_{2} @ \mathrm{MOS}$ nanosheets have excellent biocompatibility. 


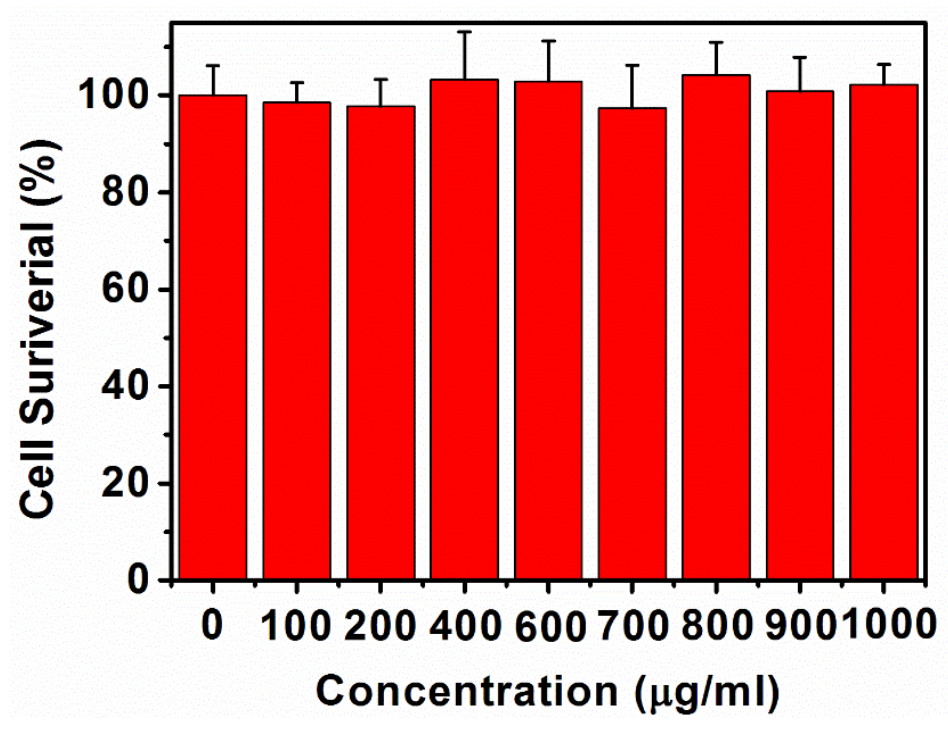

Figure 4. MCF-7 cell viability after incubating with different concentrations of the sandwich-like $\mathrm{MoS}_{2} @ \mathrm{MOS}$ nanosheets for $24 \mathrm{~h}$.

The photo-thermal performance of the sandwich-like $\mathrm{MoS}_{2} @ \mathrm{MOS}$ nanosheets was further investigated. Obviously, the temperature of the aqueous solution is increased with the $\mathrm{MoS}_{2} @ \mathrm{MOS}$ concentrations (Figure 5A). The temperature of the $\mathrm{MoS}_{2} @ \mathrm{MOS}$ solution with a concentration of $1 \mathrm{mg} \mathrm{mL}^{-1}$ rapidly increases to $44.1{ }^{\circ} \mathrm{C}$ after irradiation at $5 \mathrm{~W} \mathrm{~cm}{ }^{-2}$ for $60 \mathrm{~s}$, then reaches to $65.7{ }^{\circ} \mathrm{C}$ at $600 \mathrm{~s}$. Moreover, 0.125, 0.25 and $0.5 \mathrm{mg} \mathrm{mL} \mathrm{m}^{-1}$ of $\mathrm{MoS}_{2} @ \mathrm{MOS}$ nanosheets raised the temperatures to $40.2{ }^{\circ} \mathrm{C}, 45.4{ }^{\circ} \mathrm{C}$ and $54.6{ }^{\circ} \mathrm{C}$ at $600 \mathrm{~s}$, respectively. When decreasing the power density of the $808 \mathrm{~nm}$ laser at $2 \mathrm{~W} \mathrm{~cm}^{-2}$ (Figure S2), the temperature of $1 \mathrm{mg} \mathrm{mL}^{-1}$ of $\mathrm{MoS}_{2} @ \mathrm{MOS}$ nanosheets increased to $33.6{ }^{\circ} \mathrm{C}$ at $60 \mathrm{~s}$, then reached $44.4{ }^{\circ} \mathrm{C}$ at $600 \mathrm{~s}$ (Figure S3). All these results demonstrate that the $\mathrm{MoS}_{2} @ \mathrm{MOS}$ nanosheets has a photo-thermal transformation capability, which can be used for thermal treatment of cancer ${ }^{[30]}$ Furthermore, the photo-thermally responsive drug release property of the 
$\mathrm{MoS}_{2} @ \mathrm{MOS}$ nanosheets is investigated. In order to evaluate the stimulus drug release of DOX loaded $\mathrm{MoS}_{2} @$ MOS nanosheets, the drug release curves were measured with or without laser irradiation. Around $7.34 \%$ of DOX was release upon laser irradiation at $5 \mathrm{~W} \mathrm{~cm}^{-2}$ for $60 \mathrm{~min}$ whereas less than $1.22 \%$ leaked out without irradiation (Figure 5B). The results confirm that the $\mathrm{MoS}_{2} @ \mathrm{MOS}$ nanosheets possess photo-thermally responsive drug release property.
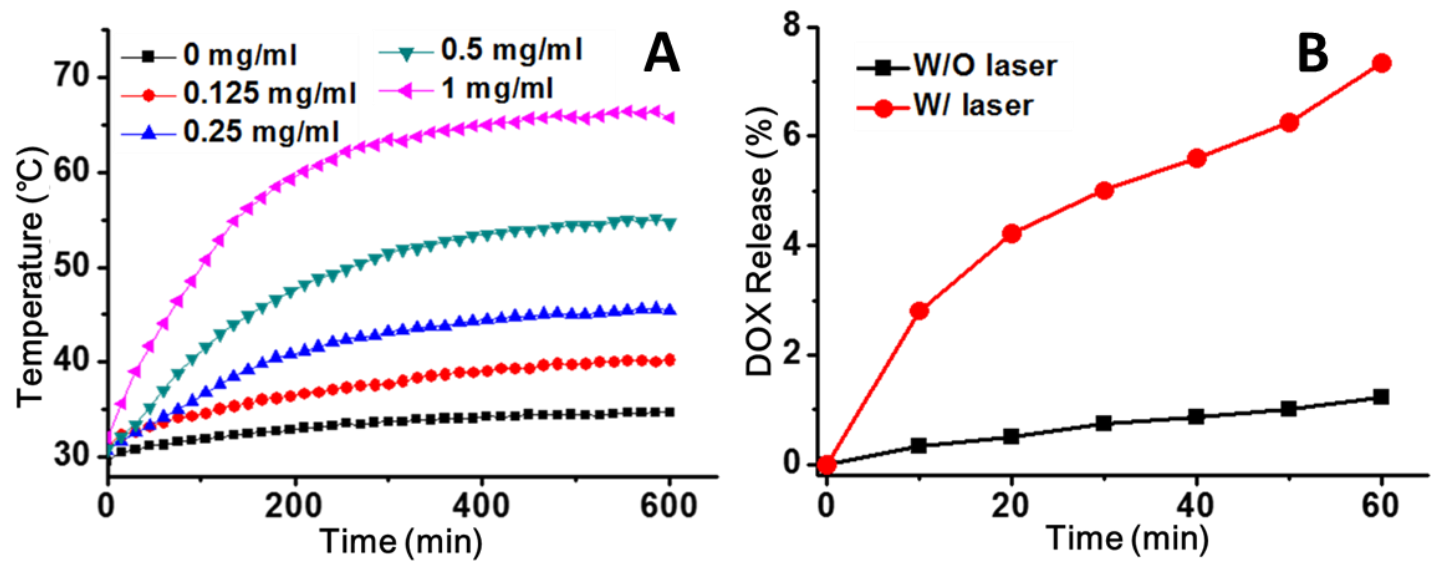

Figure 5. (A) Photo-thermal curves of the $\mathrm{MoS}_{2} @ \mathrm{MOS}$ nanosheets irradiated with a power density of $5 \mathrm{~W} \mathrm{~cm} \mathrm{~cm}^{-2}$ for $5 \mathrm{~min}$; (B) DOX release profiles from DOX/ $\mathrm{MoS}_{2} @ \mathrm{MOS}$ with or without laser irradiation.

\section{Conclusions}

In summary, well-dispersed sandwich-like $\mathrm{MoS}_{2} @ \mathrm{MOS}$ nanosheets have been first prepared by directly growth of mesostructured organosilica on $\mathrm{MoS}_{2}$ nanosheets. The resultant $\mathrm{MoS}_{2} @ \mathrm{MOS}$ nanosheets possess an ordered mesopores $(\sim 4.2 \mathrm{~nm})$, high surface area $\left(\sim 920 \mathrm{~cm}^{2} / \mathrm{g}\right)$, and large pore volume $\left(\sim 1.41 \mathrm{~cm}^{3} \mathrm{~g}^{-1}\right)$. Besides, the sandwich-like $\mathrm{MoS}_{2} @ \mathrm{MOS}$ nanosheets have excellent biocompatibility, photo-thermal conversion capability and photo-thermally controlled drug release 
property. It is believed that such novel nanosheets provide a promising strategy for cancer treatment.

\section{Acknowledgements}

We greatly appreciate financial support from the National Key Basic Research Program of the PRC (2014CB744501 and 2014CB744504), the National Natural Science Foundation of China (81201175 and 21603106), the Natural Science Foundation of Jiangsu Province (BK20160017 and BK20130863), the National

Science Foundation for Post-doctoral Scientists of China (2013T60939 and 2012M521934), the Science Foundation of Nanjing University of Posts and Telecommunications (NY213045, NY214096, and NY214012).

\section{Appendix A. Supplementary material}

Supplementary data associated with this article can be found, in the online version, at http://dx.doi.org/10.1016/j.jcis.

\section{References}

[1] W. Li and D. Zhao, Chem. Commun. 49 (2013) 943.

[2] C. M. A. Parlett, K. Wilson and A. F. Lee, Chem. Soc. Rev. 42 (2013) 3876.

[3] Y. Su, Z. Teng, H. Yao, S. Wang, Y. Tian, Y. Zhang, W. Liu, W. Tian, L. Zheng, N. Lu, Q. Ni, X. Su, Y. Tang, J. Sun, Y. Liu, J. Wu, G. Yang, G. Lu and L. Zhang, ACS Appl. Mater. Interfaces 8 (2016) 17038.

[4] X. Li, L. Zhou, Y. Wei, A. El-Toni, F. Zhang and D. Zhao, J. Am. Chem. Soc. 136 (2014), 15086. 
[5] N. Lu, Y. Tian, W. Tian, P. Huang, Y.Liu, Y. Tang, C. Wang, S. Wang, Y. Su, Y. Zhang, J. Pan, Z. Teng and G. Lu, ACS Appl. Mater. Interfaces 8 (2016), 2985.

[6] J. Wu, Y. Liu, Y. Tang, S. Wang, C. Wang, Y. Li, X. Su, J. Tian, Y. Tian, J. Pan, Y.

Su, H. Zhu, Z. Teng and G. Lu, ACS Appl. Mater. Interfaces, 8 (2016), 17927.

[7] Y. Chen, H. R. Chen, S. J. Zhang, F. Chen, L. X. Zhang, J. M. Zhang, M. Zhu, H.

X. Wu, L. M. Guo, J. W. Feng and J. L. Shi, Adv. Funct. Mater. 21 (2011), 270.

[8] G. S. Song, Q. Wang, Y. Wang, G. Lv, C. Li, R. J. Zou, Z. G. Chen, Z. Y. Qin, K.

K. Huo, R. G. Hu and J. Q. Hu, Adv. Funct. Mater. 23 (2013), 4281.

[9] D. Shen, J. Yang, X. Li, L. Zhou, R. Zhang, W. Li, L. Chen, R. Wang, F. Zhang and D. Zhao, Nano Lett. 14 (2014), 923.

[10] Q. He, J. Zhang, J. Shi, Z. Zhu, L. Zhang, W. Bu, L. Guo and Y. Chen, Biomaterials 31 (2010), 1085.

[11] D. Niu, Z. Liu, Y. Li, X. Luo, J. Zhang, J. Gong and J. Shi, Adv. Mater. 26 (2014), 4947.

[12] D. Shen, L. Chen, J. Yang, R. Zhang, Y. Wei, X. Li, Wei. Li, Z. Sun, H. Zhu, A. M. Abdullah, A. Al-Enizi, A. A. Elzatahry, F. Zhang, and D. Zhao, ACS Appl. Mater. Interfaces 7 (2015) 17450.

[13] Z. Teng, S. Wang, X. Su, G. Chen, Y. Liu, Z. Luo, W. Luo, Y. Tang, H. Ju, D. Zhao and G. Lu, Adv. Mater. 26 (2014) 3741.

[14] T. Liu, S. Shi, C. Liang, S. Shen, L. Cheng, C. Wang, X. Song, S. Goel, T. E. Barnhart, W. Cai and Z. Liu,. ACS Nano 9 (2015), 950.

[15] T. Liu, C. Wang, W. Cui, H. Gong, C. Liang, X. Shi, Z. Li, B. Sun and Z. Liu, Nanoscale 6 (2014), 11219.

[16] T. Liu, C. Wang, X. Gu, H. Gong, L. Cheng, X. Shi, L. Feng, B. Sun and Z. Liu, Adv. Mater. 26 (2014), 3433.

[17] W. Z. Teo, E. L. Chng, Z. Sofer and M. Pumera, Chem. - Eur. J. 20 (2014), 9627.

[18] W. Yin, L. Yan, J. Yu, G. Tian, L. Zhou, X. Zheng, X. Zhang, Y. Yong, J. Li, Z. Gu and Y. Zhao,. ACS Nano 8 (2014), 6922.

[19] S. S. Chou, B. Kaehr, J. Kim, B. M. Foley, M. De, P. E. Hopkins, J. Huang, C. J. Brinker and V. P. Dravid, Angew. Chem., Int. Ed. 52 (2013), 4160. 
[20] S. S. Chou, M. De, J. Kim, S. Byun, C. Dykstra, J. Yu, J. Huang and V. P. Dravid, J. Am. Chem. Soc. 135 (2013), 4584.

[21] L. Zhou, B. He, Y. Yang and Y. He, RSC Adv. 4 (2014), 32570.

[22] J. Lee, H. Park and W. J. Kim, Small 11 (2015), 5315.

[23] P. Joensen, R. F. Frindt and S. R. Morrison, Mater. Res. Bull.,1986, 21, 457.

[24] Li. Yuwen, F. Xu, B. Xue, Z. Luo, Q. Zhang, B. Bao, S. Su, L. Weng, W. Huang and L. Wang, Nanoscale 6 (2014), 5762.

[25] X. Ma, J. Zhang, M. Dang, J. Wang, Z. Tu, L. Yuwen, G. Chen, X. Su and Z. Teng, J. Colloid Interface Sci., 475 (2016), 66.

[26] Z. Teng, X. Su, B. Lee, C. Huang, Y. Liu, S. Wang, J. Wu, P. Xu, J. Sun, D. Shen, W. Li and G. Lu, Chem. Mater. 26 (2014) 5980.

[27] C. T. Kresge, M. E. Leonowicz, W. J. Roth, J. C. Vartuli and J. S. Beck, Nature 359 (1992) 710.

[28] F. X. Zhu, W. Wang and H. X. Li, J. Am. Chem. Soc. 133 (2011) 11632.

[29] H. Yang, X. Han, G. Li, Z. Ma and Y. Hao, J. Phys. Chem. C 114 (2010) 22221.

[30] S. Mura, J. Nicolas and P. Couvreur, Nat. Mater. 12 (2013), 991. 


\section{Graphical Abstract}

Sandwich-like $\mathrm{MoS}_{2} @ \mathrm{MOS}$ nanosheets have been first prepared by directly growth of mesostructured organosilica on $\mathrm{MoS}_{2}$ nanosheets. The obtained $\mathrm{MoS}_{2} @ \mathrm{MOS}$ nanosheets possess excellent dispersibility, well-defined sandwich-like structure, oriented mesopores $(\sim 4.2 \mathrm{~nm})$, high surface area $\left(\sim 920 \mathrm{~cm}^{2} / \mathrm{g}\right)$, and large pore volume $\left(\sim 1.41 \mathrm{~cm}^{3} \mathrm{~g}^{-1}\right)$.

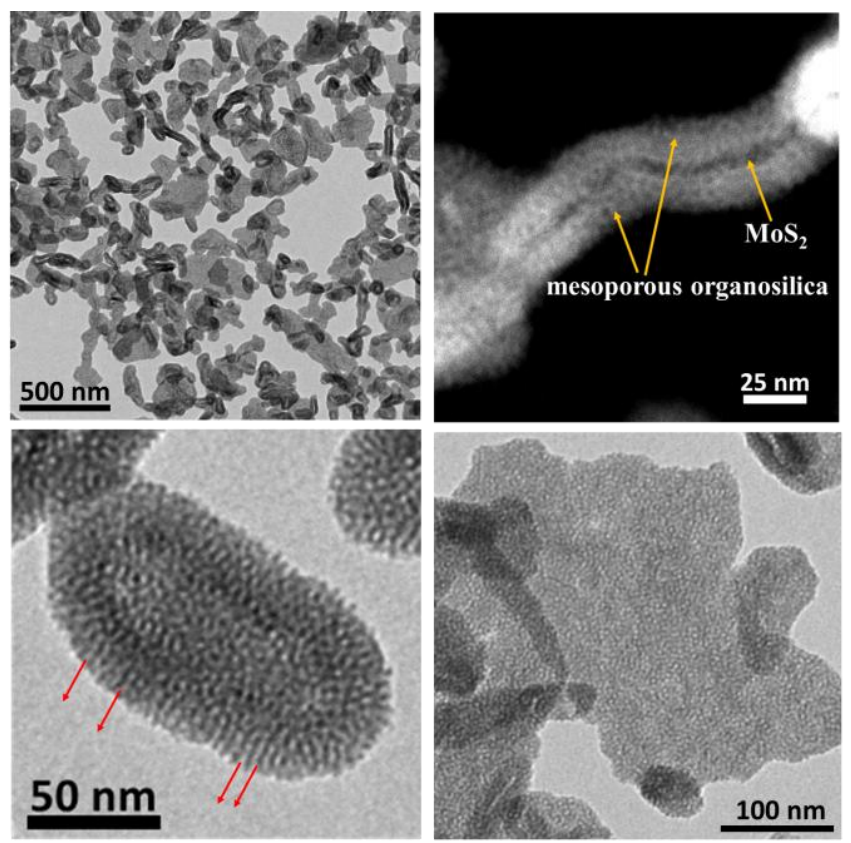

